\title{
Biología de la raya Psammobatis extenta (Garman, 1913) (Batoidea: Rajidae)
}

\author{
Biology of the skate Psammobatis extenta (Garman, 1913) (Batoidea: Rajidae)
}

\author{
JUAN M. BRACCINI ${ }^{1} \&$ GUSTAVO E. CHIARAMONTE
}

Estación Hidrobiológica de Puerto Quequén y Museo Argentino de Ciencias Naturales "Bernardino Rivadavia", Ángel Gallardo 470 (C1405DJR), Capital Federal, Argentina; e-mail: matibrac@muanbe.gov.ar',gchiaram@mail.retina.ar ${ }^{2}$

\section{RESUMEN}

Psammobatis extenta es un miembro común de la fauna de rayas de las costas de Sudamérica y su biología ha sido poco estudiada. Se investigaron aspectos de la biología de $P$. extenta a partir de datos obtenidos del descarte de arrastres comerciales de la flota pesquera de Puerto Quequén, Argentina, en febrero de 2000. La talla máxima alcanzada por las hembras fue $313 \mathrm{~mm} \mathrm{~L}_{\mathrm{T}}$ y $295 \mathrm{~mm} \mathrm{~L}_{\mathrm{T}}$, por los machos. Las proporciones que presentaron menor variación y relación isométrica fueron: distancia narina-boca, distancia interorbital, longitud ojo+espiráculo, largo de disco y ancho de disco. La relación $\mathrm{L}_{\mathrm{T}}-\mathrm{Peso}$ total presentó diferencias significativas $(\mathrm{P}<0,05)$ entre hembras y machos, las primeras alcanzando un mayor peso para el mismo $\mathrm{L}_{\mathrm{T}}$. Los machos alcanzan la madurez a un $\mathrm{L}_{\mathrm{T}}$ de $260-266 \mathrm{~mm}$, o al $89 \%$ de su talla máxima. Las hembras alcanzan la madurez a un $\mathrm{L}_{\mathrm{T}}$ de $236-255 \mathrm{~mm}$, o al $79 \%$ de su talla máxima. Las hembras grávidas (13\% de las hembras maduras) presentaron un huevo por oviducto (oviparidad simple). Psammobatis extenta presenta dimorfismo sexual en la dentición y espinas alares: los machos maduros tienen cúspides cónicas y agudas y poseen espinas alares en los márgenes exteriores del disco, mientras que las hembras y los machos inmaduros tienen dientes redondeados y romos.

Palabras clave: Psammobatis, Rajidae, Puerto Quequén.

\section{ABSTRACT}

Psammobatis extenta is a common off-shore member of the South American skate fauna and its biology is poorly known. Aspects of the biology of $P$. extenta were investigated using data collected from bycatch of commercial trawls in February 2000 off the coast of Puerto Quequén, Argentina. The maximum size attained by females was $313 \mathrm{~mm} \mathrm{~T}$ and $295 \mathrm{~mm} \mathrm{~T}_{\mathrm{L}}$ by males. Measurements that presented less variation and isometric relation were: nare to mouth length, distance between orbits, orbit and spiracle length, disc length and disc width. There were significant differences $(\mathrm{P}<$ 0.05 ) in the $T_{L}$-Total weight relation between females and males, the former attaining a greater weight for the same $\mathrm{T}_{\mathrm{L}}$. Males reach maturity between 260 and $266 \mathrm{~mm}$ in length, about $89 \%$ of their maximum size. Females reach maturity at a length of 236-255 mm, about $79 \%$ of their maximum size. Gravid females (13\% of the mature females) presented one egg case per oviduct (single oviparity). There is sexual dimorphism in dentition and alar spines: mature males have pointed conical cusps and possess alar spines along the outer margins of the upper disc, whereas female and immature males have grinding blunt teeth.

Key words: Psammobatis, Rajidae, Puerto Quequén.

\section{INTRODUCCIÓN}

En Argentina, los batoideos comprenden 38 especies pertenecientes a seis familias, de las cuales la familia cosmopolita Rajidae, es la más importante por el número (Menni 1972, Menni \& Stehmann 2000). Stehmann (1970), señala que la familia Rajidae es un grupo difícil y complejo desde el punto de vista sistemático. En la Argentina está representada por 22 especies pertenecientes a siete de los 15 géneros en que el grupo se divide (Cousseau et al. 2000). Díaz de Astarloa et al.
(1999), durante una campaña de investigación de las asociaciones ícticas a lo largo de la plataforma continental de Uruguay y norte de Argentina (34 a $41^{\circ} \mathrm{S}$ ), encontraron que la familia Rajidae presentó el mayor número de especies $(n=10$ especies).

Las especies del género Psammobatis Günther 1870 , son miembros comunes y endémicos de la fauna de rayas de las costas de Sudamérica, distribuyéndose desde el Atlántico sudoccidental hasta el Pacífico sudoriental. Menni \& Stehmann (2000) ubican a $P$. extenta (Garman) 1913 en el 
grupo de rayas que designan como bonaerenses, junto con P. bergi Marini 1932, P. rutrum Jordan 1890 y $P$. lentiginosa McEachran 1983. Según Carvalho \& de Figueiredo (1994), este género presenta especies muy similares entre sí y la determinación específica es difícil. Las proporciones morfométricas de $P$. extenta dadas en su descripción y las tomadas posteriormente por Norman (1937) y McEachran (1983), se basan en pocos ejemplares y en un rango de tallas poco representativo. Según McEachran (1983), $P$. glansdissimilis McEachran, 1983 (= P. extenta) se localiza en profundidades de hasta $160 \mathrm{~m}$, y según lo hallado por Díaz de Astarloa et al. (1999) para la plataforma continental de Uruguay y norte de Argentina, se encontró en un rango acotado de salinidad $(32,24-33,97$ ups $)$ y de temperatura $(9,6-$ $\left.12,9^{\circ} \mathrm{C}\right)$. Su distribución va desde Cabo Frío en Brasil (22 56' S) hasta los $45^{\circ} \mathrm{S}$ (Norman 1937) en Patagonia, Argentina.

Tradicionalmente, las descripciones de las especies de rayas se realizaban sobre la base de caracteres de la morfología externa como la forma del cuerpo, el tamaño, la espinulación, la coloración, la forma y cantidad de dientes y el hábitat. En general la identificación específica ha sido difícil porque un gran número de caracteres no estaba bien definido, pudiendo cambiar intraespecíficamente en relación con el tamaño de los ejemplares, con el sexo o la localización geográfica (Leible 1988).

Psammobatis extenta, uno de cuyos caracteres diagnósticos es la presencia de espínulas en el hipopilo del pterigopodio (McEachran 1983), es comúnmente capturada en los lances de pesca costera de arrastre de fondo en el litoral bonaerense y descartada (Tamini 2001), debido a que no es una especie destinada al consumo humano. Esta pesca incidental podría llegar a tener un impacto importante en su población, ya que los elasmobranquios, salvo excepciones, tienen tasas de crecimiento y reproducción lentas, que los hacen muy susceptibles a la sobreexplotación (Walker 1998). De P. extenta se conocen una aproximación a su alimentación (Soares et al. 1992) para el sur de Brasil, mientras que la presencia de dimorfismo sexual con respecto al margen anterior del disco -más curvado en las hembras y más hendido en los machos-fue notada por Norman (1937) y McEachran (1983) encontró que para todo el género, el escapulocoracoides es más corto y triangular en los machos y más rectangular en las hembras. El presente trabajo pretende aportar al conocimiento de la biología de $P$. extenta y dar parámetros morfométricos que permitan la mejor identificación de la especie.

\section{MATERIALES Y MÉTODOS}

Se analizó un total de 100 especímenes de $P$. extenta (31 machos y 69 hembras) procedentes del descarte de la pesca costera de arrastre de fondo en el área del Puerto Quequén ( $38^{\circ} 37^{\prime} \mathrm{S}$, $58^{\circ} 50^{\prime}$ O) durante el mes de febrero de 2000. Las redes fueron arrastradas por períodos de dos horas a una velocidad promedio de 2,7 nudos, generalmente sobre fondos arenosos y fangosos. Cada ejemplar fue congelado y luego sexado, pesado y medido en laboratorio. Las mediciones tomadas basándose en Leible (1988) fueron estandarizadas como porcentaje del largo total $\left(\mathrm{L}_{\mathrm{T}}\right)$, medido en los casos en que las dos aletas dorsales y la caudal se hallaron presentes. Para determinar las variables más apropiadas para la identificación de la especie se analizó el dimorfismo sexual de 13 variables morfométricas mediante una prueba $t$ de Student, eliminándose aquellas que fueron significativamente diferentes y se estudió el tipo de relación de estas variables con el $\mathrm{L}_{\mathrm{T}}$ a través de las pendientes de las regresiones lineales: alometría $(b \neq 0)$ o isometría $(b=0) ; b=$ pendiente de la regresión.

Se determinó la distribución de frecuencias $\mathrm{L}_{\mathrm{T}}$ para machos y hembras y la relación $\mathrm{L}_{\mathrm{T}}$-peso total. Para evaluar posibles diferencias entre machos y hembras en el incremento del peso en función del $\mathrm{L}_{\mathrm{T}}$ se realizó una prueba t de Student para las pendientes de las regresiones del logaritmo del peso total en función del logaritmo del $\mathrm{L}_{\mathrm{T}}$.

Con el fin de identificar cambios en el estado reproductivo de los machos con el aumento de tamaño se midieron las siguientes variables: el largo interno del pterigopodio $\left(\mathrm{L}_{\mathrm{IP}}\right)$, su grado de calcificación y la capacidad de rotación de la base hacia delante (Clark \& von Schmidt 1965); se midió el largo y el ancho de los testículos en conjunto con el órgano epigonal y se los pesó. En las hembras se registraron cualitativamente las características de los ovarios, se los pesó y se midió el ancho de las glándulas nidamentales. Se observó la presencia de huevos en los oviductos, su número y el largo, el ancho y el peso de cada uno de ellos. Finalmente y para ambos sexos, se observó a ojo descubierto el estado de desarrollo de todas las estructuras que conforman el aparato reproductor. Con toda esta información se construyó finalmente una escala de estado reproductivo (ER) para cada sexo (Tabla 1).

Para determinar diferencias en la dentición, a cada ejemplar se le extrajo la mandíbula, se contó el número de hileras de dientes y se observó bajo lupa la morfología de los mismos. 
TABLA 1

Estados reproductivos para machos y hembras de $P$. extenta

Reproductive stages for $P$. extenta males and females

\begin{tabular}{lll}
\hline Estado & \multicolumn{1}{c}{ Machos } & \multicolumn{1}{c}{ Hembras } \\
\hline I & $\begin{array}{l}\text { Testículos filiformes no mensurables, } \\
\text { formados por tejido blanco, poco } \\
\text { diferenciado. Pterigopodio blando, } \\
\text { pequeño y no calcificado }\end{array}$ & $\begin{array}{l}\text { Ovarios formados por tejido blanco, } \\
\text { poco diferenciado. Las glándulas } \\
\text { nidamentales son sólo un } \\
\text { abultamiento de los oviductos }\end{array}$ \\
II $\quad \begin{array}{l}\text { Testículos mensurables, el pterigopodio } \\
\text { comienza a alargarse pero no está } \\
\text { calcificado. Los vasos eferentes } \\
\text { comienzan a ensancharse y enrollarse } \\
\text { y la vesícula seminal, a desarrollarse }\end{array}$ & $\begin{array}{l}\text { Ovarios con ovas pequeñas y opacas. } \\
\text { Glándulas nidamentales y oviducto } \\
\text { en desarrollo }\end{array}$ \\
$\begin{array}{l}\text { Pterigopodio completamente calcificado } \\
\text { y duro. Testículos totalmente } \\
\text { desarrollados. Vasos eferentes } \\
\text { enrollados y desarrollados, vesícula } \\
\text { seminal bien desarrollada }\end{array}$ & $\begin{array}{l}\text { Ovarios completamente desarrollados, } \\
\text { llenos de ovas amarillas. Glándulas } \\
\text { nidamentales y oviductos totalmente } \\
\text { desarrollados y expandidos. } \\
\text { Cloaca amplia y expandida }\end{array}$ \\
\hline
\end{tabular}

\section{RESULTADOS}

\section{Morfometría}

Las tallas de los machos variaron entre 111-295 $\mathrm{mm} \mathrm{L}_{\mathrm{T}}$, con una mayor frecuencia entre 240-250 $\mathrm{mm} \mathrm{L} \mathrm{L}_{\mathrm{T}}$ mientras que las de las hembras lo hicieron entre 147-313 $\mathrm{mm} \mathrm{L}_{\mathrm{T}}$, con una mayor frecuencia entre 250-260 mm (Fig. 1). En la Tabla 2A se muestran las proporciones que no presentaron diferencias significativas entre machos y hembras $(\mathrm{P}>0,05)$. Entre estas variables, las que mostraron menor variación e isometría con la $\mathrm{L}_{\mathrm{T}}$ $(b=0 ; P>0,05)$ fueron: la distancia narina-boca, la distancia interorbital interna, la longitud ojo+espiráculo, la longitud del disco y el ancho del disco. En la Tabla 2B se dan las proporciones que presentaron diferencias significativas entre machos y hembras $(P<0,05)$, entre las que el ancho de boca además presenta isometría en machos y alometría en hembras. Otras variables alométricas fueron la distancia interespiracular (Tabla 2A) y longitud prenasal (Tabla 2B).

En la Fig. 2A se observa la relación $\mathrm{L}_{\mathrm{T}}$-peso para machos y hembras, expresada por la siguiente ecuación: Peso $=7 \times 10^{-6} \mathrm{x} \mathrm{L}_{\mathrm{T}}^{2,96}(\odot)$ y Peso $=2$ $\mathrm{x} 10^{-4} \mathrm{xL}_{\mathrm{T}}^{2,33}(\mathrm{)})$. Al analizar las pendientes de las regresiones de los logaritmos (Fig. 2B), se encontraron diferencias significativas entre machos y hembras $\left(\mathrm{t}_{96}=3,575, \mathrm{P}=0,00055\right)$. La pendiente de la curva de las hembras (log peso $=2,81 \mathrm{x} \log$ $\left.\mathrm{L}_{\mathrm{T}}-4,76 ; \mathrm{R}^{2}=0,86\right)$ fue más empinada que la de los machos $\left(\log\right.$ peso $=1,98 \times \log \mathrm{L}_{\mathrm{T}}-2,80 ; \mathrm{R}^{2}=$
$0,83)$, implicando que las hembras -a partir de $235 \mathrm{~mm} \mathrm{~L}_{\mathrm{T}}-$ son más robustas que los machos para una misma talla.

\section{Madurez y reproducción}

En los machos, el $\mathrm{L}_{\text {IP }}$ presentó un crecimiento de forma sigmoidal con el $\mathrm{L}_{\mathrm{T}}$ (Fig. 3A). La mayor tasa de cambio en el $\mathrm{L}_{\mathrm{IP}}$ se observó entre los 220 y $250 \mathrm{~mm} \mathrm{~L}$, indicando el rápido crecimiento en el $\mathrm{L}_{\mathrm{IP}}$ durante la maduración. De acuerdo a lo definido en la Tabla 1, el rango de los machos

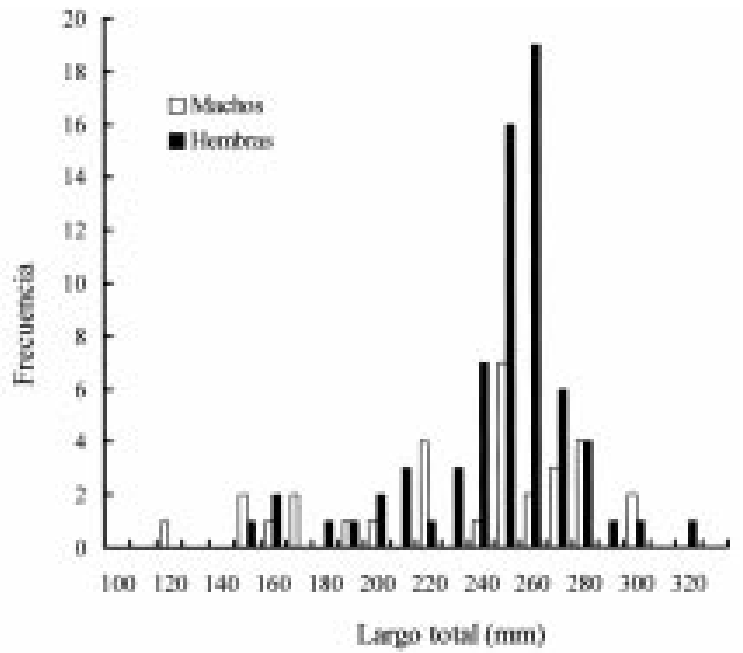

Fig. 1: Distribución de frecuencias de tallas de machos y hembras.

Length-frequency distribution for males and females. 

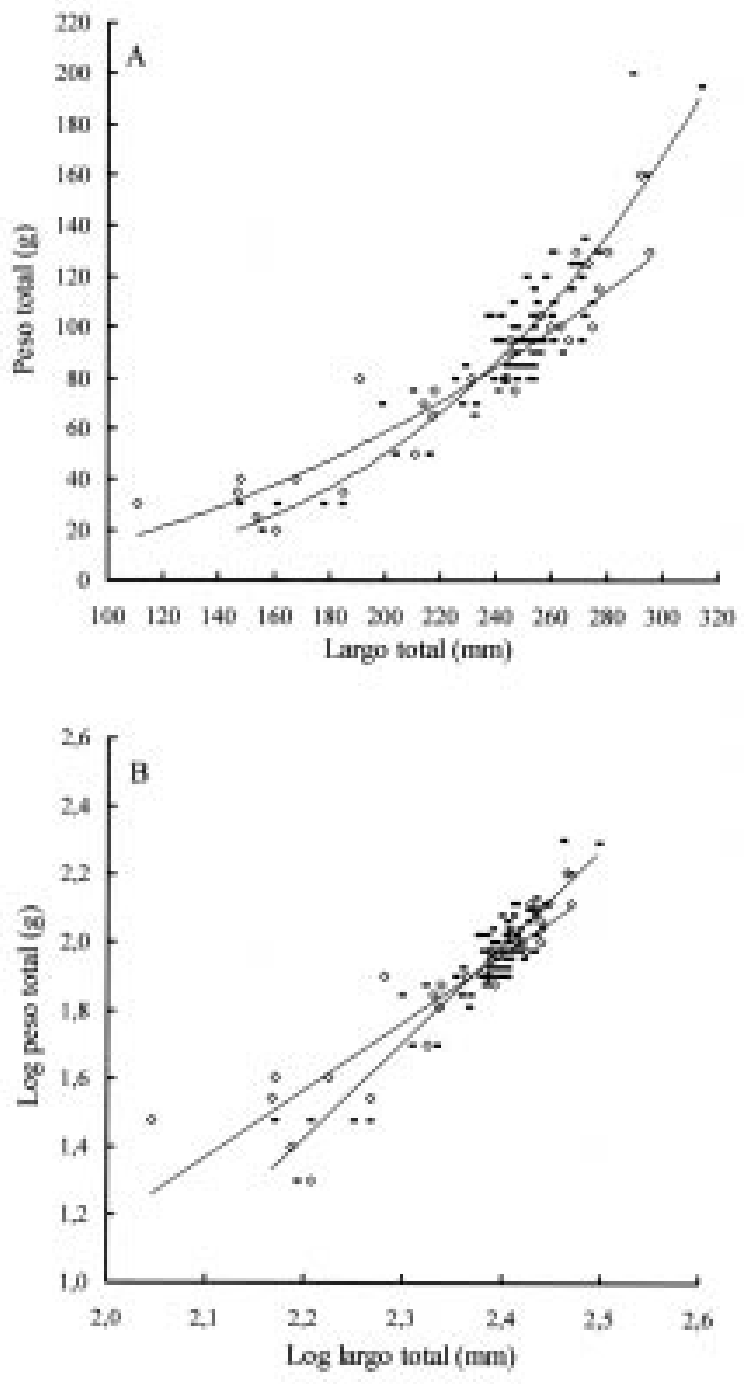

Fig. 2: (A) Relación entre el peso total y el $\mathrm{L}_{\mathrm{T}}$; (B) Regresión del logaritmo del peso total en función del logaritmo del $\mathrm{L}_{\mathrm{T}}$ para machos $(\circlearrowleft) \mathrm{y}$ hembras $(+)$.

(A) Relationship between total weight and $\mathrm{T}_{\mathrm{L}}$; (B) Regression of $T_{L}$ logarithm versus total weight logarithm for males $(\circlearrowleft)$ and females $(\varnothing)$.

correspondientes al ER I fue de 147-231, al ER II, 227-266 y al ER III, 260-295 $\mathrm{mm} \mathrm{L}_{\mathrm{T}}$. Las dimensiones de los testículos (largo y ancho) aumentaron con el $\mathrm{L}_{\mathrm{T}}$ (Fig. 3B). No existieron diferencias significativas en la longitud de los testículos izquierdo y derecho $\left(\mathrm{t}_{38}=0,006 ; \mathrm{P}=0,99\right)$ ni en su ancho $\left(t_{34}=0,008, P=0,93\right)$. Hubo un incremento en la masa gonadal con el $\mathrm{L}_{\mathrm{T}}$ (Fig. 3C). El ejemplar maduro más pequeño fue de $260 \mathrm{~mm} \mathrm{~L}_{\mathrm{T}}$. La talla de $50 \%$ de madurez sexual en los machos se dio entre los 260-269 $\mathrm{mm} \mathrm{L}_{\mathrm{T}}$ (Tabla 3). La talla de madurez sexual $(\sim 263 \mathrm{~mm})$ para los machos se
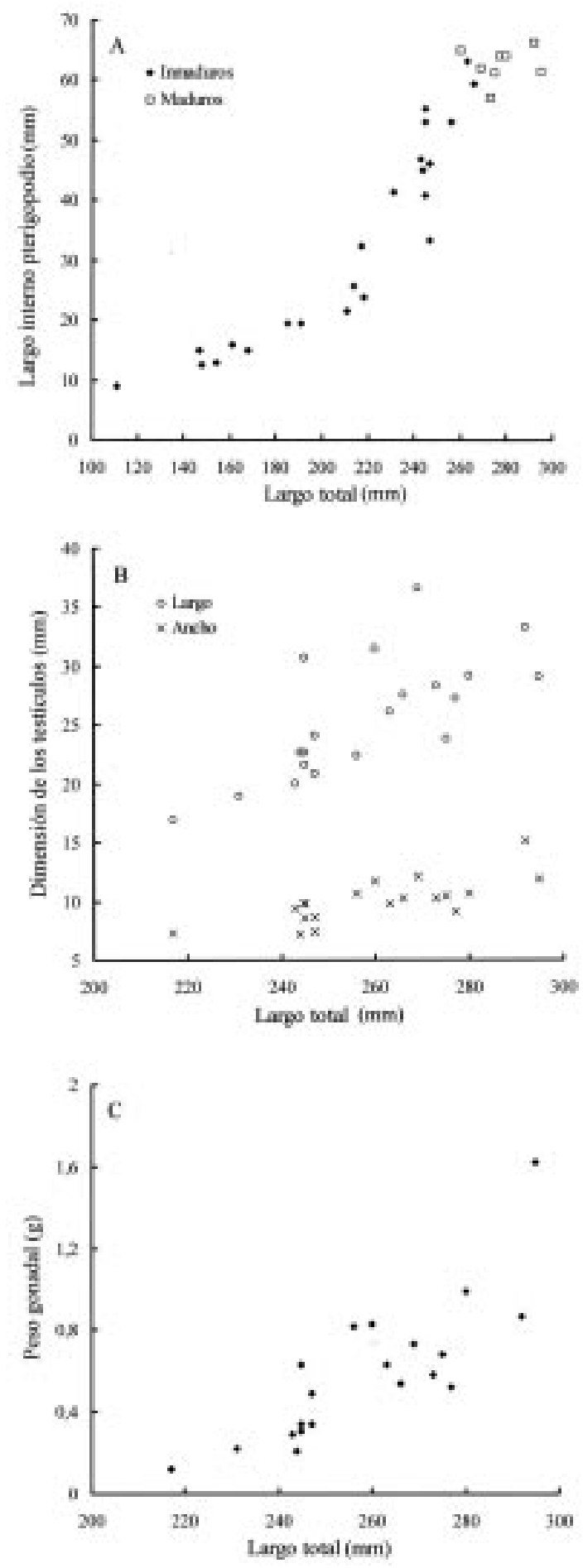

Fig. 3: Estado reproductivo de los machos: (A) relación entre el $\mathrm{L}_{\mathrm{IP}}$ y el $\mathrm{L}_{\mathrm{T}}$; (B) relación entre las dimensiones de los testículos y el $\mathrm{L}_{\mathrm{T}}$; (C) Relación entre el peso gonadal y el $\mathrm{L}_{\mathrm{T}}$.

Reproductive stage in males: (A) relationship between $\mathrm{C}_{\mathrm{IL}}$ and $\mathrm{T}_{\mathrm{L}}$; (B) relationship between testis dimensions and $\mathrm{T}_{\mathrm{L}}$; (C) relationship between gonadal weight and $\mathrm{T}_{\mathrm{L}}$. 
dio al $89 \%$ de la talla del macho más grande medido en este estudio $\left(295 \mathrm{~mm} \mathrm{~L}_{\mathrm{T}}\right)$.

En las hembras, la maduración fue caracterizada por un ensanchamiento de la glándula nidamental, la relación entre el $\mathrm{L}_{\mathrm{T}}$ y el ancho de la glándula nidamental presentó un mayor crecimiento entre los 240 y $260 \mathrm{~mm} \mathrm{~L}_{\mathrm{T}}$ (Fig. 4A). De acuerdo a lo definido en la Tabla 1, el rango de las hembras correspondientes al ER I fue de $147-$ 245, al ER II, 198-255 y al ER III, 236-313 mm $\mathrm{L}_{\mathrm{T}}$. La masa gonadal también presentó un incremento con el $\mathrm{L}_{\mathrm{T}}$, con un crecimiento máximo entre 240 y $260 \mathrm{~mm} \mathrm{~L}_{\mathrm{T}}$ (Fig. 4B). Las hembras adultas tuvieron un ancho máximo de ovas entre 5,2-16 mm. El ejemplar adulto de menor tamaño fue de $236 \mathrm{~mm}$. E1 $50 \%$ de madurez sexual en las hembras fue entre 236-255 $\mathrm{mm} \mathrm{L}_{\mathrm{T}}$ (Tabla 3), presentando un abrupto alargamiento de la glándula nidamental a partir de ese rango de $\mathrm{L}_{\mathrm{T}}$ (Fig. 4A). La talla de madurez sexual ( $246 \mathrm{~mm})$ para las hembras se dio al $79 \%$ de la talla de la hembra más grande medida en este estudio $\left(313 \mathrm{~mm} \mathrm{~L}_{\mathrm{T}}\right)$. El $13 \%$ de las hembras adultas (entre 236 y 313 $\mathrm{mm} \mathrm{L}_{\mathrm{T}}$ ) presentó un huevo por oviducto. Los huevos fueron de color marrón claro con largos zarcillos en cada esquina. Su longitud media fue de $24,19 \mathrm{~mm}$ (rango 15,1-29,3) y su ancho medio fue de 17,77 mm (rango 13,7-21,1). Las hembras que llevaban huevos además poseían ovas maduras (ancho máximo entre 6,4-13,7 mm) en sus ovarios.

\section{Espinas alares}

En los machos, a partir de $241 \mathrm{~mm} \mathrm{~L}_{\mathrm{T}}$ se observó el desarrollo de aguijones o espinas en la región alar dispuestos en columnas en sentido anteroposterior, con sus ápices apuntando hacia el centro del disco. En ejemplares hasta $251 \mathrm{~mm}$ $\mathrm{L}_{\mathrm{T}}$ se contó una sola columna de aguijones ocupando sendas cavidades epidérmicas, sin sobresalir; entre $255-282 \mathrm{~mm} \mathrm{~L}_{\mathrm{T}}$ una sola columna de aguijones ya emergidos de las cavidades; entre 279-297 $\mathrm{mm} \mathrm{L}_{\mathrm{T}}$ dos columnas, y en un ejemplar de $311 \mathrm{~mm} \mathrm{~L}_{\mathrm{T}}$, capturado en una muestra no analizada, se observaron tres columnas.

En las hembras, a partir de $229 \mathrm{~mm} \mathrm{~L}_{\mathrm{T}}$ se observó el desarrollo de aguijones o espinas en la región alar dispuestos sin un patrón definido, con sus ápices apuntando hacia el borde posterior del disco.

TABLA 2

Variables medidas estandarizadas como porcentaje del $\mathrm{L}_{\mathrm{T}}$ (en $\left.\mathrm{mm}\right)$ : (A) no dimórficas (P > $0,05), \mathrm{n}=100 ;(\mathrm{B})$ dimórficas $(\mathrm{P}<0,05)$, machos $\mathrm{n}=31$, hembras $\mathrm{n}=69$

Proportional measurements in percent of $\mathrm{T}_{\mathrm{L}}($ in $\mathrm{mm})$ : $(\mathrm{A})$ non-dimorphic $(\mathrm{P}>0.05), \mathrm{n}=100 ;(\mathrm{B})$ dimorphic $(\mathrm{P}<$ $0.05)$, males $\mathrm{n}=31$, females $\mathrm{n}=69$

(A)

\begin{tabular}{|c|c|c|c|c|}
\hline Variable & Relación & $\overline{\mathrm{X}} \pm \mathrm{DE}$ & Rango & Valor de $\mathrm{P}$ \\
\hline Longitud total & & $238,88 \pm 36,77$ & $111-313$ & 0,11 \\
\hline Longitud disco & Isométrica & $47,62 \pm 1,71$ & $43,67-53,45$ & 0,14 \\
\hline Ancho disco & Isométrica & $57,31 \pm 1,73$ & $53,23-61,53$ & 0,9 \\
\hline Longitud ojo + espiráculo & Isométrica & $4,69 \pm 0,53$ & $3,83-7,00$ & 0,31 \\
\hline Distancia interespiracular & Alométrica & $6,81 \pm 0,42$ & $5,07-8,46$ & 0,78 \\
\hline Distancia interorbital interna & Isométrica & $4,03 \pm 0,34$ & $3,25-4,94$ & 0,71 \\
\hline Distancia narina-boca & Isométrica & $1,93 \pm 0,33$ & $1,21-3,47$ & 0,54 \\
\hline
\end{tabular}

(B)

\begin{tabular}{|c|c|c|c|c|c|c|}
\hline \multirow[t]{2}{*}{ Variable } & \multirow{2}{*}{ Relación } & \multicolumn{2}{|c|}{ Machos } & \multicolumn{2}{|c|}{ Hembras } & \multirow[b]{2}{*}{ Valor de $\mathrm{P}$} \\
\hline & & $\bar{X} \pm D E$ & Rango & $\bar{X} \pm D E$ & Rango & \\
\hline Distancia hocico-axila & Isométrica & $40,83 \pm 1,03$ & $38,77-43,24$ & $42,07 \pm 1,48$ & $36,82-46,18$ & $<0,0001$ \\
\hline Longitud hocico & Isométrica & $12,34 \pm 0,76$ & $10,90-14,14$ & $12,75 \pm 0,71$ & $11,21-15,02$ & 0,01 \\
\hline Longitud prenasal & Alométrica & $8,77 \pm 0,83$ & $6,85-10,63$ & $9,37 \pm 0,52$ & $8,17-10,58$ & 0,0005 \\
\hline Distancia internasal & Isométrica & $3,59 \pm 0,33$ & $2,96-4,34$ & $3,43 \pm 0,32$ & $2,82-4,43$ & 0,03 \\
\hline Ancho boca & $*$ & $6,03 \pm 0,67$ & $4,91-7,75$ & $5,50 \pm 0,54$ & $4,48-7,17$ & 0,0003 \\
\hline Distancia hocico-cloaca & Isométrica & $41,29 \pm 0,48$ & $40,36-41,93$ & $43,14 \pm 1,30$ & $39,25-47,89$ & 0,03 \\
\hline Longitud cola & Isométrica & $57,96 \pm 1,07$ & $56,26-60,24$ & $55,81 \pm 1,39$ & $51,34-60,06$ & $<0,0001$ \\
\hline
\end{tabular}

*Alométrica en hembras e isométrica en machos 


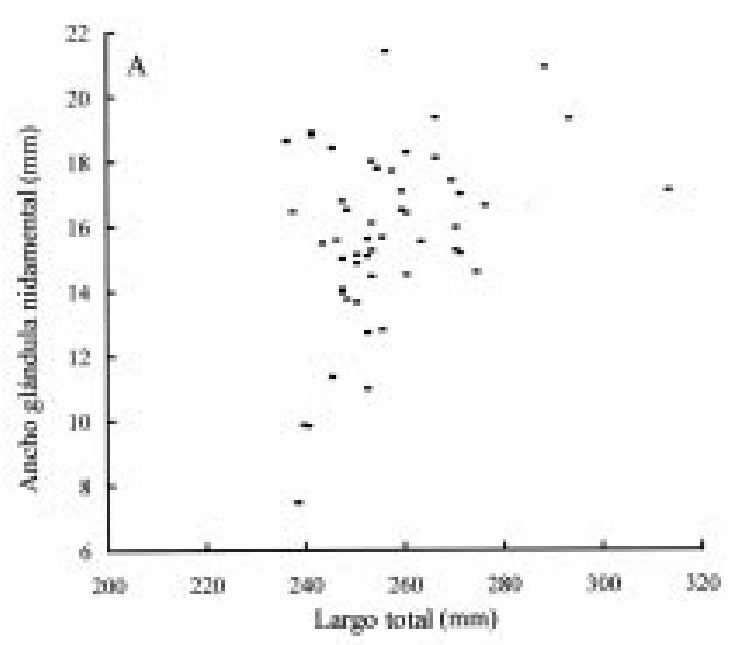

\section{Dentición}

En los machos se contó entre 34-45 hileras de dientes en la mandíbula superior y entre 34-44 en la inferior. En las hembras se observaron entre 35-46 hileras en la mandíbula superior y entre 34-46 en la inferior.

Los machos juveniles y las hembras de P. extenta presentaron dientes de forma redondeada con la punta cónica y roma, distribuidos en hileras oblicuas (Fig. 5A y 5B). Por su parte, los machos maduros presentaron dientes de base redondeada y forma cónica, con su punta aguda, en hileras longitudinales (Fig. 5C y 5D).

\section{DISCUSIÓN}

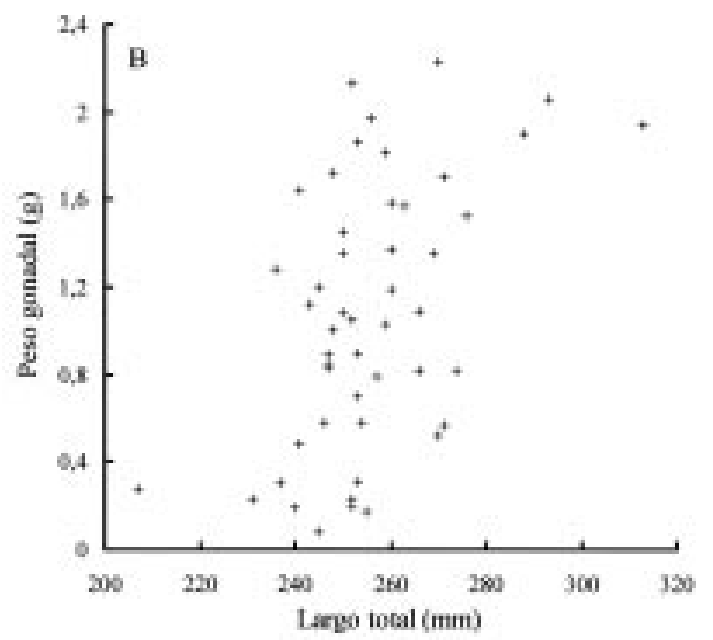

Fig. 4: Estado reproductivo de las hembras: (A) relación entre el ancho de la glándula nidamental y el $\mathrm{L}_{\mathrm{T}}$; (B) relación entre el peso gonadal y el $\mathrm{L}_{\mathrm{T}}$. Reproductive stage in females: (A) Relationship between shell gland width and $\mathrm{L}_{\mathrm{T}}$; (B) Relationship between gonadal weight and $T_{L}$.

En taxonomía es esencial elegir características que varíen poco dentro de un grupo o taxón, ya que son más confiables que las altamente variables para la comparación taxonómica (Bass 1973). La medición de estructuras externas y las proporciones y relaciones obtenidas de las mismas han sido parte de la sistemática de elasmobranquios desde mediados del siglo pasado hasta el presente. Springer (1964) discutió las dificultades de tomar mediciones sobre ejemplares preservados, mientras que Bass (1973) y Bass et al. (1973, $1975 \mathrm{a}, 1975 \mathrm{~b})$ analizaron las variaciones proporcionales y cambios de éstas con el crecimiento en un buen número de especies de tiburones. Bass (1973) propone tres tipos diferentes de regresiones para las variables morfométricas estandarizadas respecto del $\mathrm{L}_{\mathrm{T}}$ : constante, lineal y curvilínea. Sólo las constantes o isométricas permitirían caracterizar a una especie.

Norman (1937) da las proporciones morfométricas de $P$. extenta sobre la base de tres especímenes. McEachran (1983), además de trabajar con 33 variables morfométricas externas en 27 ejemplares, incluye otras variables de la anatomía del neurocráneo y del pterigopodio. Carvalho (1991) ${ }^{1}$, cuando revisó críticamente los

TABLA 3

Porcentaje de individuos maduros a un determinado $\mathrm{L}_{\mathrm{T}} \mathrm{y}$ número de especímenes analizados

Percent of mature individuals at a given $\mathrm{T}_{\mathrm{L}}$ and number of specimens sampled

\begin{tabular}{lccccc}
\hline & $\begin{array}{c}\text { Machos } \\
\mathrm{L}_{\mathrm{T}}(\mathrm{mm})\end{array}$ & Número de especímenes & Madurez $(\%)$ & $\mathrm{L}_{\mathrm{T}}(\mathrm{mm})$ & $\begin{array}{c}\text { Hembras } \\
\text { Número de especímenes }\end{array}$ \\
\hline $111-259$ & 21 & 0 & $147-235$ & 14 & 0 \\
$260-269$ & 4 & 50 & $236-245$ & 12 & 50 \\
$270+$ & 6 & 100 & $246-255$ & 24 & 79 \\
& & & $256+$ & 19 & 100 \\
\hline
\end{tabular}



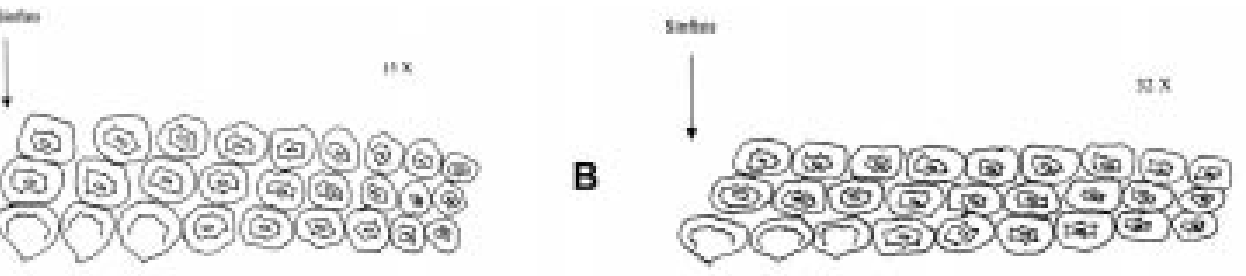
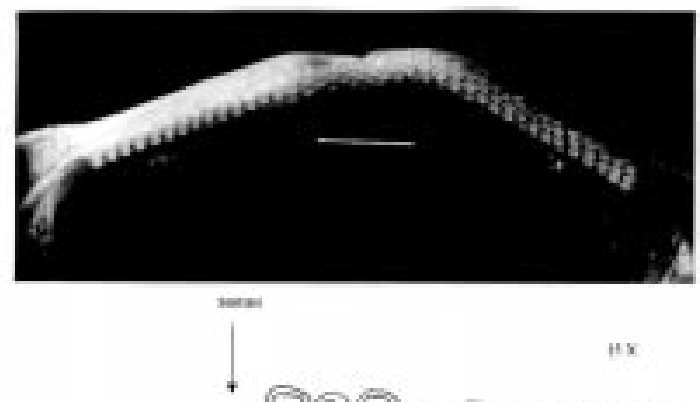

C

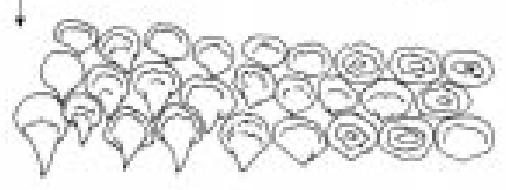

D

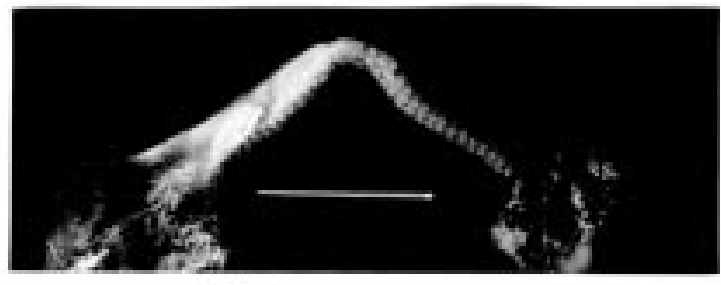

$\operatorname{set}$
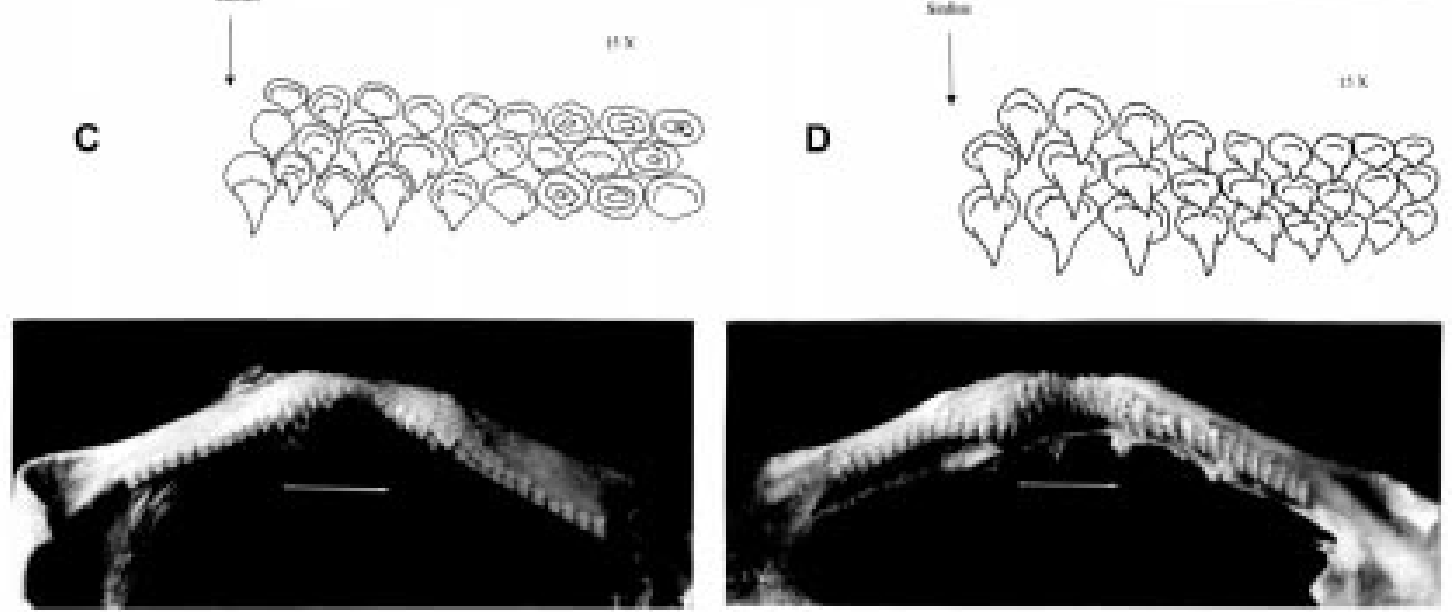

Fig. 5: Dimorfismo sexual en dientes: hembras maduras y machos inmaduros con dentición similar y cambios en machos maduros durante el desarrollo. En el extremo superior se representan las primeras nueve hileras de dientes de la mandíbula superior desde la sínfisis hacia la izquierda: (A) hembra madura de $313 \mathrm{~mm} \mathrm{~L}_{\mathrm{T}}$; (B) macho inmaduro de $161 \mathrm{~mm} \mathrm{~L}_{\mathrm{T}}$; (C) macho maduro de $266 \mathrm{~mm} \mathrm{~L}_{\mathrm{T}}$; (D) macho maduro de $295 \mathrm{~mm} \mathrm{~L}_{\mathrm{T}}$. Escala: $5 \mathrm{~mm}$.

Teeth sexual dimorphism: mature females and immature males with similar dentition and changes in mature males' development. At the top are represented the first nine teeth rows of the superior jaw from the symphysis to the left: (A) mature female $313 \mathrm{~mm} \mathrm{~T}_{\mathrm{L}}$; (B) immature male $161 \mathrm{~mm} \mathrm{~T}_{\mathrm{L}}$; (C) mature male $266 \mathrm{~mm} \mathrm{~T}$; (D) mature male $295 \mathrm{~mm} \mathrm{~T}_{\mathrm{L}}$. Scale bar: $5 \mathrm{~mm}$.

caracteres diagnósticos, estableció que la altura de la aleta caudal es más baja que la altura de la segunda dorsal y que la distancia interorbital es mayor que el diámetro orbital, y subsecuentemente Carvalho \& Figueiredo (1994), consideraron a $P$. glansdissimilis un sinónimo junior de $P$. extenta. En la muestra analizada de Puerto Quequén se pudieron discriminar sólo diez medidas propor-

${ }^{1}$ DE CARVALHO MR (1991) Nova chave para identificar as especies de Psammobatis Gunther, 1870 (Rajidae) con notas sobre a sua posiçao filogenetica e biogeografia. Resumen V Reunião Grupo de Trabalho Pesca e Pesquisa Tubarões e Arraias no Brasil, Santos, São Paulo: pp. 7. cionales isométricas para ambos sexos (Tabla 2). El estudio de las variaciones de las mismas fue posible gracias al número de ejemplares de la muestra, y aunque a diferencia del trabajo de McEachran (1983), se trabajó con material fresco, varias de las proporciones analizadas coincidieron con las expuestas por este autor.

Si bien Lamilla et al. (1984), estudiando la biología de Sympterigia lima (Poeppig) 1835, encontraron que la pesca comercial del litoral de Valdivia fue selectiva para esa especie por las artes de pesca utilizadas, el arte utilizado por la flota costera con base en Puerto Quequén no ejerció una presión de selectividad en las tallas, 
ya que los lances de pesca duraron ca. $2 \mathrm{~h}$, colmatándose las redes y perdiendo su selectividad. Por esta razón se puede suponer que las tallas más frecuentes para machos y hembras no estarían sesgadas por el arte de pesca.

Walmsley-Hart et al. (1999) encontraron que las tasas de crecimiento de Leucoraja wallacei (Hulley) 1970 y Dipturus pullopunctatus (Smith) 1964 difirieron entre sexos, un rasgo común en las especies de Rajidae; las hembras alcanzan un tamaño mayor que los machos, pero crecen más lentamente, en concordancia con la generalidad dada en elasmobranquios, donde las hembras alcanzan una mayor talla y peso corporal que los machos. Los machos de P. extenta presentaron un menor peso corporal que las hembras para una misma talla, a partir de $\sim 235 \mathrm{~mm} \mathrm{~L}_{\mathrm{T}}$, siendo esta relación significativamente diferente.

En los elasmobranquios, la determinación de la madurez sexual de los machos se fundamenta en la dureza y desarrollo del pterigopodio (Pratt 1988); así, los Rajidae exhiben una abrupta transición en la longitud del pterigopodio con relación a la madurez sexual, similar a otros batoideos (Smith \& Merriner 1987). Psammobatis extenta no es la excepción: basados en el rápido alargamiento de pterigopodio y calcificación completa, y la presencia de testículos, vasos eferentes y vesícula seminal totalmente desarrollados, el rango de madurez sexual en los machos se determinó entre 260-266 $\mathrm{mm} \mathrm{L}_{\mathrm{T}}$. La talla de madurez sexual ( $263 \mathrm{~mm}$ ) para los machos se dio al $89 \%$ del macho más grande medido en este estudio (295 mm). Zeiner \& Wolf (1993) encontraron que para los machos de Raja binoculata Girard 1854, la madurez sexual se dio al 57-72 \% (1000-1100 $\mathrm{mm}$ ) de la $\mathrm{L}_{\mathrm{T}}$ asintótica calculada, mientras que los machos de Dipturus rhina (Jordan \& Gilbert) 1880 lo hicieron al $62 \%(600 \mathrm{~mm})$ de $1 \mathrm{a} \mathrm{L}_{\mathrm{T}}$ asintótica calculada.

En las hembras, en cambio, la determinación de la madurez sexual se basó en las siguientes evidencias: el rápido crecimiento en ancho de la glándula nidamental, alcanzando los $14 \mathrm{~mm} \mathrm{o}$ más, la presencia de ovas amarillas en los ovarios, de oviductos completamente desarrollados y de una cloaca expandida y amplia, registrándose el rango de madurez en las hembras entre 236$255 \mathrm{~mm} \mathrm{~L}_{\mathrm{T}}$. La talla de madurez sexual ( 246 $\mathrm{mm}$ ) para las hembras se dio al $72 \%$ de la hembra más grande medida en este estudio (313 $\mathrm{mm})$. Zeiner \& Wolf (1993) encontraron que para las hembras de $R$. binoculata, la madurez sexual se dio al $82 \%(1300 \mathrm{~mm})$ de la $\mathrm{L}_{\mathrm{T}}$ asintótica calculada, mientras que las hembras de $D$. rhina 10 hicieron al $66 \%(700 \mathrm{~mm})$ de la $\mathrm{L}_{\mathrm{T}}$ asintótica calculada. El $13 \%$ de las hembras adultas conte- nía huevos, presentando oviparidad simple, llevando un huevo por oviducto cada una; además presentaron ovas maduras por lo que se puede inferir que $P$. extenta podría volver a aparearse inmediatamente después de depositar los huevos o fecundar ovocitos con esperma almacenado de una sola cópula anual.

Según Walmsley-Hart et al. (1999), el mayor tamaño corporal alcanzado por las hembras de Rajidae es probablemente una consecuencia de sus diferentes estrategias reproductivas; las hembras logran un mayor tamaño para poder llevar los huevos dentro de la cavidad del cuerpo y los machos crecen más rápidamente para alcanzar la madurez sexual. Aunque estos autores no estudiaron si el ancho de disco presenta dimorfismo sexual, en su trabajo concluyen que los machos de L. wallacei maduraron a un ancho de disco menor que las hembras. Este patrón también fue encontrado por Zeiner \& Wolf (1993); los machos de $R$. binoculata y D. rhina maduraron antes (7-8 años en $R$. binoculata y 5 años en $D$. rhina) que las hembras (12 años en $R$. binoculata y 8 años en $D$. rhina). En los ejemplares de $P$. extenta analizados, las hembras maduraron a una menor $\mathrm{L}_{\mathrm{T}}$ que los machos, y la hembra adulta más pequeña fue de $24 \mathrm{~mm}$ de $\mathrm{L}_{\mathrm{T}}$ menor que el macho adulto más pequeño $\left(=236 \mathrm{~mm} \mathrm{~L}\right.$ 的 $\left.\sigma=260 \mathrm{~mm} \mathrm{~L}_{\mathrm{T}}\right)$, coincidiendo con la talla $(\sim 235 \mathrm{~mm})$ a partir de la cual las hembras presentaron un mayor peso corporal que los machos. Quizás porque la talla de $P$. extenta es menor que las de las especies citadas por Walmsley-Hart et al. (1999) y el peso de las hembras crece más rápido que el de los machos (Fig. 2A), esta especie podría presentar una estrategia reproductiva distinta a la comúnmente encontrada en los elasmobranquios.

A diferencia de Lamilla et al. (1984), quienes encontraron dimorfismo sexual en el ancho del disco de $S$. lima (más ancho en las hembras que en los machos de la misma talla), en P. extenta no se registró este tipo de dimorfismo. En los organismos en los que hay dimorfismo sexual podría existir una utilización diferencial del nicho por los distintos sexos, que conduciría a una menor competencia intraespecífica por el alimento (Feduccia \& Slaughter 1974). Estos autores sostuvieron que dentro de los Rajidae, las especies son sexualmente dimórficas o no dimórficas, e interpretaron la existencia de dimorfismo sexual en el aparato de masticación como una forma de hacer posible el forrajeo diferencial entre machos y hembras, disminuyendo la competencia intraespecífica por el alimento. Por otra parte, en una interpretación más plausible, McEachran (1976) propone que el dimorfismo sexual es más importante en el comportamiento reproductivo 
que en la diferenciación de utilización del nicho. Si el dimorfismo de los dientes está relacionado con la diferenciación del nicho, debería darse previamente y no recién en la maduración sexual (McEachran 1976). Por ejemplo, Lamilla et al. (1984), encontraron diferencias en la dentición de machos y hembras de $S$. lima, pero no así en su dieta, que es similar en ambos sexos. Según Breder \& Rosen (1966), durante la cópula los machos usan sus dientes y espinas alares para sujetar a la hembra. McEachran \& Musick (1973), hacen notar que la aparición de dientes con punta en los machos coincide con la aparición de aguijones o espinas alares. Si bien no se ha observado dimorfismo sexual en el número de hileras de dientes, en los machos maduros de $P$. extenta, los dientes se asemejaron a las espinas del lado dorsal del disco, concordando así con la hipótesis de que las distintas formas dentarias podrían ser de mayor importancia en el comportamiento reproductivo. Otras variables, en este caso morfométricas (Tabla 2B), también presentaron dimorfismo sexual, siendo estos hechos aún de difícil interpretación.

Los elasmobranquios presentan un crecimiento lento (Beamish \& McFarlane 1985) y las rayas no son una excepción; esta característica, al combinarse con estrategias reproductivas que producen pocos juveniles bien desarrollados, debe ser considerada al evaluar el impacto potencial que el descarte puede llegar a tener en las poblaciones locales de rayas (Walmsley-Hart et al. 1999). Por otra parte, Walker \& Heessen (1996), encontraron una relación entre el aumento en la densidad de la especie no comercial Amblyraja radiata (Donovan) 1807, entre 1970 y 1999, y la virtual desaparición de la especie comercial Raja clavata Linn 1758, desde 1958. Tomando en cuenta estos antecedentes, concluimos que se debe profundizar el estudio del conjunto de rayas presentes en la zona para poder evaluar el modo en que la pesca comercial de algunas especies de rayas actúa sobre las especies descartadas. En particular, es necesario elucidar el ciclo reproductivo y la fecundidad relativa, que en el caso de $P$. extenta podría no ser estacional y por lo tanto continua a lo largo del año. De esta manera, esta especie estaría afectada en menor medida por la pesquería que otras especies de condrictios en los que la tasa de fecundidad es baja y estacional.

\section{AGRADECIMIENTOS}

Agradecemos a L. Tamini, y a A.J. Alarcos por el apoyo en el trabajo de campo. A patrones y tripulaciones de los B/P Angela, B/P Angel Antonio, $\mathrm{B} / \mathrm{P}$ Assunta, B/P Dele dele, B/P Eusonia, B/P
Felicitas, B/P Jesús de Nazareth, B/P La Cruz del Sud y B/P La Cruz del Sur. R. Bruno, J. Renaudo y personal de Pescadería Santa Cecilia. A Prefectura Naval Argentina, destacamento Puerto Quequén. A G. Pastorino por el trabajo fotográfico. A R.C. Menni por la revisión crítica y enriquecedores comentarios del manuscrito. Este trabajo se realizó en el marco del subsidio otorgado a L. Cappozzo por la Fundación Antorchas (Proyecto A-13672/1-3).

\section{LITERATURA CITADA}

BASS AJ (1973) Analysis and description of variation in the proportional dimensions of scyliorhinid, carcharhinid and sphyrnid sharks. South African Association for Marine Biological Research, Oceanographic Research Institute, Investigational Report No. 32. 28 pp.

BASS AJ, JD D'AUBREY \& N KISTNASAMY (1973) Sharks of the east coast of southern Africa. I. The genus Carcharhinus (Carcharhinidae). Investigational Report of Oceanographic Research Institute (Durban) 33: 1-168.

BASS AJ, JD D'AUBREY \& N KISTNASAMY (1975a) Sharks of the east coast of southern Africa. II. The families Scyliorhinidae and Pseudotriakidae. Investigational Report of Oceanographic Research Institute (Durban) 37: 1-63.

BASS AJ, JD D'AUBREY \& N KISTNASAMY (1975b) Sharks of the east coast of southern Africa. III. The families Carcharhinidae (excluding Mustelus and Carcharhinus). Investigational Report of Oceanographic Research Institute (Durban) 38: 1-100.

BEAMISH RJ \& GA MCFARLANE (1985) Annulus development on the second dorsal spine of the spiny dogfish (Squalus acanthias) and its validity for age determination. Canadian Journal of Fisheries and Aquatic Science 42: 1799-1805.

BREDER CM \& DE ROSEN (1966) Modes of reproduction in fishes. Natural History Press, Garden City, New York. 941 pp.

CARVALHO MR DE \& JL DE FIGUEIREDO (1994) Psammobatis extenta (Garman, 1913): a senior synonym of Psammobatis glansdissimilis McEachran, 1983 (Chondrichthyes, Rajidae). Copeia 1994: 10291033.

CLARK E \& K VON SCHMIDT (1965) Sharks of the Central Gulf Coast of Florida. Bulletin of Marine Science 15: 13-83.

COUSSEAU MB, DE FIGUEROA \& JM DÍAS DE ASTARLOA (2000) Clave de identificación de las rayas del litoral marítimo de Argentina y Uruguay (Chondricthyes, Familia Rajidae). Instituto Nacional de Investigación y Desarrollo Pesquero (INIDEP), Mar del Plata, Argentina. 35 pp.

DÍAZ DE ASTARLOA JM, A AUBONE \& MB COSSEAU (1999) Asociaciones ícticas de la plataforma costera de Uruguay y norte de Argentina, y su relación con los parámetros ambientales. Physis (Argentina)A 57: $29-45$. 
FEDUCCIA A \& BH SLAUGHTER (1974) Sexual dimorphism in skates (Rajidae) and its possible role in differential niche utilization. Evolution 28: 164168.

GARMAN SW (1913) The Plagiostomia (sharks, skates and rays). Memoirs of Harvard Museum of Comparative Zoologie 36: 1-528.

LAMILLA JG, G PEQUEÑO \& H FIGUEROA (1984) Aspectos biológicos de Psammobatis lima, Poeppig, 1835, en el litoral de Valdivia, Chile (Elasmobranchii, Rajidae). Revista de la Comisión Permanente del Pacífico Sur 14: 183-209.

LEIBLE MD (1988) Revisión de métodos para estudios taxonómicos de rayas (Rajiformes, Rajidae). Gayana Zoología (Chile) 52: 15-93.

MCEACHRAN JD (1976) Reply to: "Sexual dimorphism in skates (Rajidae)". Evolution 31: 218-220.

MCEACHRAN JD (1983) Results of the research cruises of FRV "Walther Herwig" to South America. LXI. Revision of the South American skate genus Psammobatis Günther, 1870. (Elasmobranchii: Rajiformes, Rajidae). Archiv für Fischeriwissenschaf Bundesforschungsanstalt für Fischerie 34: 23-80.

MCEACHRAN JD \& JA MUSICK (1973) Characters for distinguishing between immature Specimens of the sibling species, Raja erinacea and Raja ocellata (Pisces: Rajidae). Copeia 1973: 238-250.

MENNI RC (1972) Raja (Atlantoraja) subgen. nov. y lista crítica de los Rajidae argentinos (Chondrichthyes, Rajiformes). Revista del Museo de La Plata (nueva serie), Zoología 11: 165-173.

MENNI RC \& MFW STEHMANN (2000) Distribution, environment and biology of batoid fishes off Argentina, Uruguay and Brazil. A review. Revista del Museo Argentino de Ciencias Naturales (nueva serie) 2: 69-109.

NORMAN JR (1937) Coast fishes, Part. II. The Patagonian region. Discovery Reports 16: 1-150.

PRATT HL (1988) Elasmobranch gonad structure: a description and survey. Copeia 1988: 719-729.

SMITH JW \& JV MERRINER (1987) Age and growth, movements and distribution of the cownose ray, Rhinoptera bonasus in Chesapeake Bay. Estuaries 10: 153-164.
SOARES L SH, CL ROSSI-WONGTSCHOWSKI, LMC ALVARES, E MUTO \& ML GASALLA (1992) Grupos tróficos de peixes demersais da plataforma continental interna de Ubatuba, Brasil. I. Chondrichthyes. Boletim Instituto Oceanografico de São Paulo 40: 7985.

SPRINGER VG (1964) A revision of the carcharinid shark genera Scoliodon, Loxodon and Rhizoprionodon. Proceedings of the United States National Museum 115: 559-632.

STEHMANN M (1970) Vergleichend morphologische und anatomische untersuchunger zur Neuordnung der Systematik der nordostatlantischen Rajidae (Chondrichthyes, Batoidei). Archiv für Fischeriwissenschaf Bundesforschungsanstalt für Fischerie 21: 73-164.

TAMINI LL (2001) Estudio del descarte en la pesca de arrastre de fondo en el área de Puerto Quequén, Provincia de Buenos Aires, Argentina. Tesis de Licenciatura, Facultad de Ciencias Exactas y Naturales, Universidad de Buenos Aires, Buenos Aires, Argentina. $63 \mathrm{pp}$.

WALKER PA \& HJL HEESSEN (1996) Long-term changes in ray populations in the North Sea. ICES Journal of Marine Science 53: 1085-1093.

WALKER TI (1998) Can shark resources be harvested sustainably? A question revisited with a review of shark fisheries. Marine and Freshwater Research 49: 553-572.

WALMSLEY-HART SA, WHH SAUER \& CD BUXTON (1999) The biology of the skates Raja wallace $i$ and $R$. pullopunctata (Batoidea: Rajidae) on the Agulhas Bank, South Africa. South African Journal of Marine Science 21: 165-179.

ZEINER SJ \& P WOLF (1993) Growth characteristics and estimates of age at maturity of two species of skates (Raja binoculata and Raja rhina) from Monterey Bay, California. Conservation Biology of Elasmobranchs: 87-99. NOAA Technical Report NMFS 115. 